TITLE:

\title{
Apex of a V-shaped cut field acts as a pacemaker on an oscillatory system
}

\section{$\operatorname{AUTHOR}(\mathrm{S})$ :}

Motoike, Ikuko N.; Nakata, Satoshi; Iguchi, Yasutaka; Takemura, Kaori K.; Hayashi, Kumiko; Yoshikawa, Kenichi

\section{CITATION:}

Motoike, Ikuko N....[et al]. Apex of a V-shaped cut field acts as a pacemaker on an oscillatory system. Chemical Physics Letters 2010, 490(4-6): 238-241

\section{ISSUE DATE:}

2010-04-26

URL:

http://hdl.handle.net/2433/120963

\section{RIGHT:}

(c) 2010 Elsevier B.V.; この論文は出版社版でありません。引用の際には 出版社版をご確認ご利用ください。; This is not the published version. Please cite only the published version. 


\title{
Apex of a V-shaped cut field acts as a pacemaker on an oscillatory system
}

\author{
Ikuko N. Motoike ${ }^{\mathrm{a}, \mathrm{b}}$, Satoshi Nakata ${ }^{\mathrm{c}, \mathrm{d}}$, Yasutaka Iguchi ${ }^{\mathrm{c}}$, Kaori K. \\ Takemura $^{\mathrm{c}}$, Kumiko Hayashi ${ }^{\mathrm{c}}$, Kenichi Yoshikawa ${ }^{\mathrm{e}, \mathrm{f}}$ \\ ${ }^{a}$ PRESTO, Japan Science and Technology Agency (JST), 4-1-8 Honcho Kawaguchi, \\ Saitama, 332-0012, Japan \\ ${ }^{b}$ Institute for Integrated Cell-Material Science (iCeMS), Kyoto University, Kyoto, \\ 606-8501, Japan \\ ${ }^{c}$ Dept. of Chemistry, Nara University of Education, Takabatake-cho, Nara, 630-8528, \\ Japan \\ ${ }^{d}$ Dept. of Mathematical and Life Sciences, Hiroshima University, Kagamiyama 1-3-1, \\ Higashi-Hiroshima, 739-8526, Japan \\ ${ }^{e}$ Dept. of Physics, Kyoto University, Kyoto, 606-8502, Japan \\ ${ }^{f}$ Spatio-Temporal Order Project, ICORP, Japan Science and Technology Agency (JST), \\ Kyoto 606-8502, Japan
}

\begin{abstract}
The generation and propagation of chemical waves in the Belousov-Zhabotinsky (BZ) reaction were investigated using a cation-exchange membrane embedded with a metal catalyst as a 2-dimensional oscillatory/active field. We found that a target pattern is generated from the top of a V-shape, indicating that the apex on the boundary between active and passive fields behaves as a pacemaker. A Plausible mechanism for this phenomenon is proposed based on a reaction-diffusion equation.
\end{abstract}

Keywords: Chemical waves, Reaction-diffusion, Belousov-Zhabotinsky (BZ) reaction, pacemaker

Email address: i.n.motoike@icems.kyoto-u.ac.jp (Ikuko N. Motoike) 


\section{Introduction}

Oscillatory and excitable chemical reactions have attracted considerable interest over the past few decades [1]. Among the many oscillatory systems described, the Belousov-Zhabotinsky (BZ) reaction has been used as the most representative oscillatory medium [1-3]. In this reaction, a concentric target pattern with circular symmetry is generated on a thin unstirred layer of the reaction medium using a pacemaker, such as a silver wire [1]. The pacemaker is at the center of oscillation, which exhibits the shortest periodicity, or the highest activity, in the oscillatory/excitable field. The spatio-temporal behavior of the chemical wave is generally described within the framework of a reaction-diffusion equation [1]:

$$
\frac{\partial \boldsymbol{u}}{\partial t}=F(\boldsymbol{u})+\boldsymbol{D} \nabla^{2} \boldsymbol{u}
$$

where $\boldsymbol{u}=\boldsymbol{u}(\boldsymbol{r}, t)$ represents the concentration variables and $\boldsymbol{D}$ is the diffusion matrix. When $F$ is a suitable nonlinear function for the reaction, Eq. (1) produces nontrivial spatio-temporal patterns in a system which is spatially uniform with respect to these parameters. In a usual aqueous solution, the diagonal elements in the diffusion matrix $\boldsymbol{D}$, or the diffusion constants of the individual chemical species, are essentially the same, since the dependence of $\boldsymbol{D}$ on $M$ is rather insensitive; i.e., $\boldsymbol{D}$ scales as $M^{-1 / 3}\left(\sim V^{-1 / 3}\right)$, where $M$ is the molecular weight of a species and $V$ is its volume. On the other hand, it has been reported that the diffusion of certain chemical components can be reduced on a suitable reaction medium, such as an ion-exchange membrane loaded with catalyst [4]. In the present study, we have examined the generation and propagation of traveling waves on a BZ medium with a 
cation-exchange membrane that was uniformly loaded with metal-catalyst, which implies the effect of decreased diffusion of the inhibitor.

\section{Experimental Section}

\subsection{Materials}

A nafion cation-exchange membrane (Aldrich, Nafion 117, perfluorinated membrane, thickness: $0.18 \mathrm{~mm}$ ) was used as the medium in the BZ reaction $[3,5]$. Stock solutions of iron (II) tris-(1, 10-phenanthroline)(ferroin), sodium bromate, sodium bromide, malonic acid (MA), and sulfuric acid were prepared with reagent-grade chemicals (Wako Pure Chemicals, Japan). The water was purified with a Millipore Milli-Q filtering system, which was maintained at $18 \mathrm{M} \Omega$.

\subsection{Procedure}

The membrane was carefully cut with a ceramic knife to obtain the desired shape. A nafion membrane doped with ferroin $\left(8.0 \mathrm{mmol} / \mathrm{m}^{2}\right)$. The reactant solution was composed of $0.4 \mathrm{M} \mathrm{KBrO}_{3}, 0.3 \mathrm{M} \mathrm{H}_{2} \mathrm{SO}_{4}, 0.02 \mathrm{M} \mathrm{NaBr}$, and 0.2 M malonic acid. The ferroin-loaded membrane was prepared by immersing the nafion membrane in $0.2 \mathrm{mM}$ ferroin aqueous solution with stirring at room temperature [5]. The bathing solution became colorless within several hours after immersion, indicating that all of the catalyst was absorbed and immobilized on the nafion membrane. The catalyst-loaded membrane was then placed in catalyst-free BZ solution at a depth of $3 \mathrm{~mm}$ to induce the chemical waves on the membrane. The membrane was in contact with the bottom surface of the reaction vessel. The chemical waves were observed 
with a digital video camera (SONY, DCR-PC7, time resolution: 1/30 s). All measurements were performed at $300 \pm 1 \mathrm{~K}$.

\subsection{Results}

Figure 1 exemplifies the generation of traveling waves in a V-shaped oscillatory field for a nafion membrane doped with ferroin, where a V-shaped $60^{\circ}$ notch has been cut from the left side. The tip of the notch acts as a pacemaker; periodic traveling waves are generated at the edge of the $\mathrm{V}$-shaped cut. This observation shows that the oscillatory medium is activated to the highest degree at this apex. Figures 1a-e show the initial stage of the experiments, with a time interval of $20 \mathrm{~s}$. Figure $1 \mathrm{f}$ shows the pattern of the chemical wave $1 \mathrm{~h}$ after the start of the experiment and indicates that the edge of the V-shaped cut still acts as a pacemaker.

We examined the effect of the angle of the $\mathrm{V}$-shaped cut on the generation of the traveling wave. When the angle of the cut was $90^{\circ}$, traveling waves were generated from the $\mathrm{V}$-shaped notch at a probability of $55 \%$ in 30 experiments. In other cases, traveling waves were generated from a random location along the edge of the strip. The probability of wave generation from the notch increased with a decrease of the angle; the probabilities of wave generation were 73 and $77 \%$ for angles of $60^{\circ}$ and $30^{\circ}$, respectively.

\section{Discussion}

Let us consider why the $\mathrm{V}$-shaped cut acts as a pacemaker. We adapt the theoretical framework of the Rovinsky-Zhabotinsky model equations $[6,7]$ : 


$$
\left\{\begin{aligned}
\frac{\partial x}{\partial \tau} & =\frac{1}{\epsilon}\left\{x(1-x)-2 q \alpha \frac{z}{1-z} \frac{x-\mu}{x+\mu}\right\}+D_{x} \nabla^{2} x \\
\frac{\partial z}{\partial \tau} & =x-\alpha \frac{z}{1-z}+D_{z} \nabla^{2} z
\end{aligned}\right.
$$

where $x$ and $z$ correspond to $\left[\mathrm{HBrO}_{2}\right]$ and $\left[\mathrm{Fe}(\mathrm{phen})_{3}^{3+}\right]$, respectively. In our experimental system, $D_{z} \approx 0$, since the catalyst is fixed on the ionexchange membrane $[5,8,9]$. To examine the effect of diffusion on the excitable/oscillatory region adjacent to the diffusion field in a simple manner, we replace $D_{x} \nabla^{2} x$ with $-c x$. With this change, the rate of the escape of the activator through the boundary is accounted for in a conventional approximation manner. With these considerations, these equations are reduced to ordinary differential equations with two variables, $x$ and $z$ :

$$
\left\{\begin{array}{l}
\frac{d x}{d \tau}=\frac{1}{\epsilon}\left\{x(1-\epsilon c-x)-2 q \alpha \frac{z}{1-z} \frac{x-\mu}{x+\mu}\right\} \equiv f(x, z) \\
\frac{d z}{d \tau}=x-\alpha \frac{z}{1-z} \equiv g(x, z)
\end{array}\right.
$$

We next address the behavior of the above system by considering the null clines: $\Sigma, f(x, z)=0$ and $\Gamma, g(x, z)=0$.

$$
\left\{\begin{aligned}
\text { nullcline } \Sigma: z & =\frac{x(x+\mu)(1-\epsilon c-x)}{2 q \alpha(x-\mu)+x(x+\mu)(1-\epsilon c-x)} \\
& \equiv h(x) \\
\text { nullcline } \Gamma: z & =\frac{x}{\alpha+x}
\end{aligned}\right.
$$

We adopt the same values for the parameters, $(q=0.5, \alpha=0.0017408$, $\epsilon=0.147059$ and $\mu=0.00051$ ) [6]. From a geometrical analysis of the phase 
plane, it is obvious that the system in Eq. (4) is oscillatory. In an oscillatory cycle, as shown in Fig. 2(a), it has been well established $[1,10,11]$ that the rate-determining process corresponds to the change from $A$ to $B$, as in Figs. 2(a) and (b). $A$ and $B$ are local maximum and minimum points, respectively, where $\partial z / \partial x=\partial h(x) / \partial x=0$ of null cline $\Sigma$ in Eq. (4). The values of $z$ at points $\mathrm{A}$ and $\mathrm{B}$ are functions of $c$. Thus, the period $T_{\mathrm{AB}}$, as a function of $c$, is given approximately by the period of this rate-determining process from Eq. (5) [1].

$$
T_{A B} \simeq \int_{z_{\mathrm{A}}}^{z_{\mathrm{B}}} \frac{1-z}{x_{\mathrm{B}}-\left(x_{\mathrm{B}}+\alpha\right) z} d z
$$

From the relationship in Eq. (5), the period $T_{\mathrm{AB}}$ can be represented as a function of $c$, as shown in Fig. 2(c). The reason on the decrease of the period owe to the escape of the activator may be explaind as follows. As in Fig.2 (a), inclusion of the escape rate caused the right branch of $\Sigma$ to shift toward the left, i.e., to move toward the smaller $x$. This induces the shrinkage of the limit cycle, implying the whole path along the limit cycle decreases. As a result, we may expect that the period $T_{\mathrm{AB}}$ decreases monotonically with an increase in $c$. Thus, the frequency increases with an increase in the escape rate of the activator, in contrast to a simple expectation. Due to above mechanism we can consider the possibility that the local region with a high frequency behaves as a pacemaker. Figure 3 shows the results of a numerical simulation for the oscillatory system in Eq. (3) with a V-shaped cut. With a spatially uniform state as the initial condition, chemical waves are generated near the edge of the $\mathrm{V}$-shaped cut.

Thus, the numerical simulation reproduces the essential behavior ob- 
served in the experiment depicted in Fig. 1. The existence of a relaxation in oscillation is believed to play a role. Relaxation oscillation is a rather general characteristic of non-linear oscillation, as in a beating heart, nerve firing, etc.

As a related past study, Bishop et al. has reported that an effect of complex geometry of the BZ system on the generation of chemical wave, under the experimental conditions where the oscillatory region was limited at border line of pentagonal geometry [12]. However, the present study indicates the clear experimental results that an apex on the boundary between active and passive fields plays a role as a pacemaker. Here, it may be of interest to notice that fibrillation in a beating heart is closely related to the existence of a local dead region or inactive spot $[13,14]$.

\section{Conclusion}

We demonstrated both experimentally and numerically on the generation and propagation of a BZ wave depending on the shape of the boundary, and particularly a V-shaped notch. We consider that the most significant result of the current paper is showing that geometrical effect on the possibility of decrease of the period accompanied with the escape of the activator. Actual periodicity is not a simple function of the escape rate of the activator but is determined by a rather complicated process on the kinetics both on activator and inhibitor, involving accumulation of diffused materials [15]. Further studies on the effect of diffusion from the reaction field into the environment may be necessary. Why the V-cut apex behaves as the pacemaker has not been clarified yet and that it may be a challenging issue to perform the numerical simulation on 2-D and/or 3-D to evaluate the effect on the periodicity 
depending on the actual geometry.

Spatial geometrical effects in excitable/oscillatory systems have also recently attracted attention as a way to realize anisotropic wave propagation in excitable/oscillatory media [15-19]. The ability to control the manner of wave propagation may be useful for constructing a signal-processing unit with excitable/oscillatory media [20-24]. Further studies on spatial geometrical effects in reaction-diffusion systems are expected to provide new perspectives on spatio-temporal structures under far-from-equilibrium conditions.

\section{Acknowledgement}

The authors thank Professor Anatol M. Zhabotinsky for providing indispensable advice during his stay in Japan, and would like to honor his achievements in the study of nonlinear phenomena. We also thank Professor Yoshihito Mori for his kind discussions and comments.

[1] R. Field, M. Burger, Oscillations and Traveling Waves in Chemical Systems, Wiley, New York, 1985.

[2] A.N. Zaikin, A.M. Zhabotinsky, Nature 225 (1970) 535-537.

[3] I.R. Epstein, K. Showalter, J. Phys. Chem. 100 (1996) 13132-13147.

[4] A. Lázár, Z. Noszticzius, H.-D. Försterling, Z. Nagy-Ungváraii, Physica D 84 (1995) 112-119.

[5] D. Winston, M. Arora, J. Maselko, V. Gáspár, K. Showalter, Nature 351 (1991) 132-135.

[6] A.B. Rovinskii, A.M. Zhabotinskii, J. Phys. Chem. 88 (1984) 6081-6084. 
[7] X.-G. Wu, S. Nakata, M. Menzinger, A. Rovinsky, J. Phys. Chem. 100 (1996) 15810-15814.

[8] A. Rovinsky, M. Menzinger, Phys. Rev. Lett. 70 (1993) 778-781.

[9] K. Yoshikawa, R. Aihara, K. Agladze, J. Phys. Chem. A 102 (1998) 7649-7652.

[10] J.J. Tyson, P.C. Fife, J. Chem. Phys. 73 (1980) 2224-2237.

[11] R.J. Field, R.M. Noyes, J. Chem. Phys. 60 (1974) 1877-1884.

[12] K.J.M. Bishop, B.A. Grzybowski, Phys. Rev. Lett. 97 (2006) 128702.

[13] K. Agladze, M.W. Kay, V. Krinsky, N. Sarvazyan, Am. J. Physiol. Heart Circ. Physiol. 293 (2007) H503-513.

[14] J. Cysyk, L. Tung, Biophys. J. 94 (2008) 1533-1541.

[15] T. Kusumi, T. Yamaguchi, R.R. Aliev, T. Amemiya, T. Ohmori, H. Hashimoto, K. Yoshikawa, Chem. Phys. Lett. 271 (1997) 355-360.

[16] K. Agladze, R.R. Aliev, T. Yamaguchi, K. Yoshikawa, J. Phys. Chem. 100 (1996) 13895-13897.

[17] Á. Tóth, D. Horváh, K. Yoshikawa, Chem. Phys. Lett. 345 (2001) 471474.

[18] T. Ichino, K. Fujio, M. Matsushita, S. Nakata, J. Phys. Chem. A 113 (2009) 2304-2308. 
[19] J.N. Gorecka, J. Gorecki, Y. Igarashi, J. Phys. Chem. A 111 (2007) 885-889.

[20] I. Motoike, K. Yoshikawa, Phys. Rev. E 59 (1999) 5354-5360.

[21] I.N. Motoike, K. Yoshikawa, Y. Iguchi, S. Nakata, Phys. Rev. E 63 (2001) 036220.

[22] Á. Tóth, K. Showalter, J. Chem. Phys. 103 (1995) 2058-2066.

[23] J. Gorecka, J. Gorecki, Phys. Rev. E 67 (2003) 067203.

[24] A. Adamatzky, B. Costello, T. Asai, Reaction-Diffusion Computers, Elsevier, Amsterdam, 2005.

[25] W. Press, B. Flannery, S. Teukolsky, W. Vetterling (Eds.), Numerical Recipes, in C: The Art of Scientific Programming, Cambridge U. Press, Cambridge, England, 1992. 


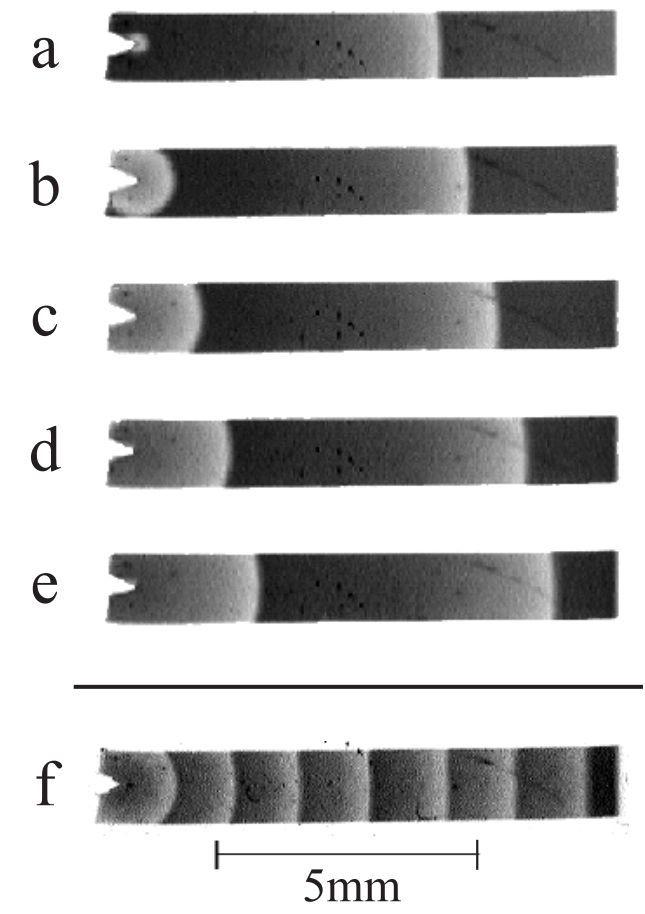

Figure 1: Generation of traveling waves from the edge of a V-shaped notch cut in a nafion membrane. The images were obtained about $5 \mathrm{~min}(\mathrm{a}-\mathrm{e})$ and $1 \mathrm{~h}$ (f) after the reaction was started. The time interval between frames in (a-e) is $20 \mathrm{~s}$. 
(a)

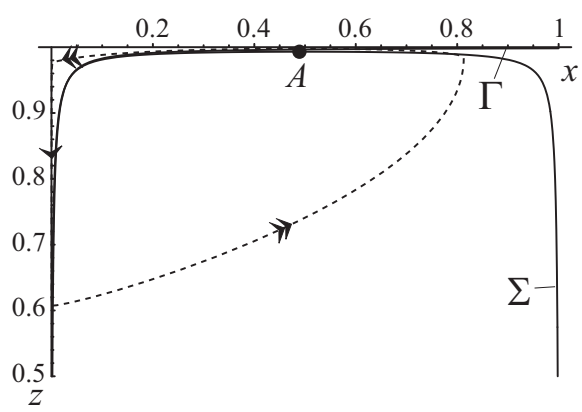

(b)

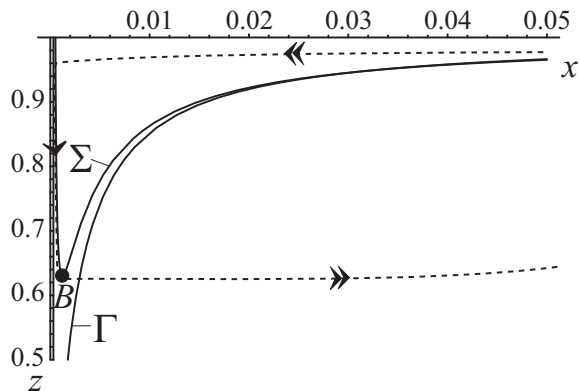

(c)

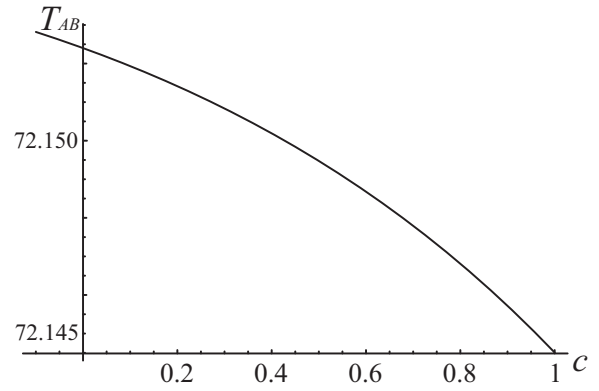

Figure 2: (a): Null clines in Eq. (4) and limit-cycle of the system in Eq.(2) at $c=0$. Null clines and limit-cycle are denoted as solid and broken lines, respectively. (b): Enlarged graph of (a). (c): Dependence of the periodicity $T_{\mathrm{AB}}$ of the oscillation on the effect of enhanced diffusion $c$. 
(a)

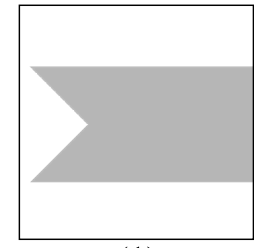

(1)

(b)

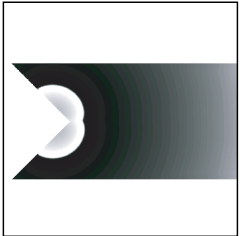

(4)

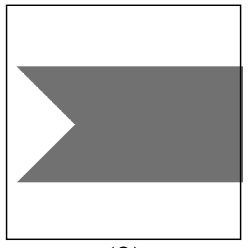

(2)

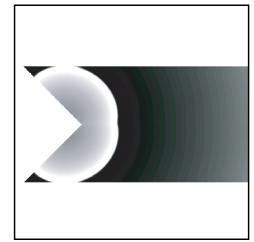

(5)

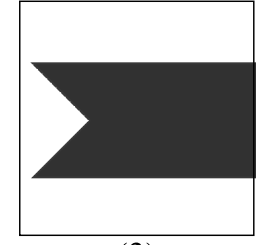

(3)

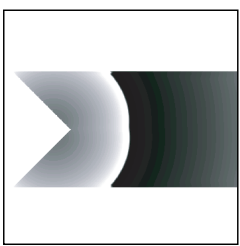

(6)

Figure 3: Theoretical calculation on the time evolution of the concentration profile of the inhibitor $z$ on a reaction-diffusion field with a V-shaped cut and an outer diffusion field. The system of partial differential Eq. (2) was calculated numerically using the ADI method (alternating direction implicit method) [25]. The parameters are $q=0.5$, $\alpha=0.0017408, \epsilon=0.147059, \mu=0.00051$, and $D_{x}=0.0181$. The grid size is $1000 \times 1000$ points in a square lattice, and the time interval is $\Delta t=0.005$, and the space interval is $\Delta x=0.05$. The boundary condition at the edge of the frame is taken to be no flux, while that between the reaction-diffusion and passive diffusion fields is taken to be free flux of activator $x$. The spatial-profile of the inhibitor $z$ in Eq. (2) is shown in quasi-color, where brighter regions correspond to a higher concentration. Figures show the concentration of the inhibitor $z$ for (a) Early stage and (b) late stage. (1) $\tau=6$, (2) $\tau=20$, (3) $\tau=42$, (4) $\tau=7065$, (5) $\tau=7073$, and (6) $\tau=7081$. 